\title{
ESCALA DE DESESPERANZA DE BECK (BHS): ADAPTACIÓN Y CARACTERÍSTICAS PSICOMÉTRICAS
}

\author{
Jaime Aliaga Tovar*, Luis Rodriguez de los Rios**, Carlos Ponce Diaz ${ }^{* * *}$,

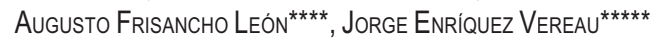

\section{RESUMEN}

En este artículo presentamos la adaptación y el estudio psicométrico de la confiabilidad y validez de la Escala de Desesperanza de Beck (BHS) en la población de Lima Metropolitana. La traducción se realizó con la técnica back translation. La muestra de estudio fue de 782 sujetos (327 varones y 445 mujeres) con edades entre 13 a 19 años (mediana $=19$ años); dividida en siete grupos: personas con intento de suicidio $(\mathrm{n}=26)$; pacientes con depresión mayor o trastorno bipolar $(n=23)$; hipertensos, asmáticos y tuberculosos $(n=68)$; pacientes esquizofrénicos paranoides y con trastornos afectivos $(n=22)$; pacientes dependientes a la pasta básica de cocaína $(n=25)$; estudiantes del 6to. grado de primaria $(n=51) ; y$, amas de casa, estudiantes del quinto de secundaria, universitarios, docentes $(n=567$; población general). El coeficiente alfa de Crombach es de $0.80(n=782)$ y el coeficiente de correlación de Pearson de los puntajes del test-retest con un intervalo de dos semanas es $0.60(n=100)$. La correlación de la BHS con los ítems que evalúan la desesperanza de la escala de depresión de Beck (BID) es de 0.46; y la correlación con la BID sin los ítemes de desesperanza es de 0.40. $\mathrm{El}$ análisis factorial con la técnica de los componentes principales y rotación varimax encuentra 6 factores que explican el $49.32 \%$ de la varianza. También se observa que el sexo no se correlaciona con la BHS, pero sí lo hace la edad $(\mathrm{p}<0.01)$. Por último, se halla que los pacientes con depresión mayor o trastorno bipolar se agrupan mayoritariamente en las categorías moderado/severo de desesperanza; y los sujetos del grupo población general; los asmáticos, hipertensos, asmáticos, así como los adictos a la PBC se agrupan mayoritariamente en las categorías Normal-asintomático/leve de desesperanza. Se concluye que la BHS adaptada evalúa un constructo psicológicamente significativo y puede ser usada con propósitos de screning.

Palabras clave: Escala de Desesperanza de Beck, Adaptación, Confiabilidad, Validez.

\begin{abstract}
ABSTRAC
In this paper we presented the adaptation and psychometric study of the Beck Hopelesness Scale (BHS). The sample consisted of 782 subjects (372 males and 445 females) The median age was 19 years. The sample this conformated by suicide attempters $(n=26)$;

\footnotetext{
* Docente Principal. Facultad de Psicología de la UNMSM. E-mail: aliato@terra.com.pe

** Docente Principal. Universidad Nacional de Educación. E-mail: vicerectoradoa@une.edu.pe

*** Docente Principal. Facultad de Psicología de la UNMSM. E-mail: cponced@unmsm.edu.pe

*** Docente Auxiliar. Facultad de Psicología de la UNMSM. E-mail: aufril56@yahoo.es

$* * * * *$ Investigador colaborador.
} 
recurrent-episode major depression and bipolar disorder patients $(n=23)$; hipertensives, asmathics and tuberculosius patients $(\mathrm{n}=68)$; paranoid schizophrenics and affective disorders patients $(n=22)$; PBC addicts $(n=25) ; 6^{\text {th }}$.grade primary students $(n=51)$; housewives, secondary and universitary students, teachers $(\mathrm{n}=567$; general population). The Alpha Crombach's was $0.80(\mathrm{~N}=782)$ and the Pearson correlation between the test-retest scores for sample $(n=100)$ who were tested at intake and two weeks later was 0.60 . The correlation between the BDI Hopelesness/Pessimism item and the BHS was 0.46; the correlation between the BDI subtracting Hopelesness/Pessimism item and the BHS was 0.40. The Factor Analysis of the items whit components principal and varimax rotation techniques found six factors explained the 49.32 of variance. The BHS score was not significantly related to sex but was positively related to age $(\mathrm{p}<0.01)$. Finally, the major depression and bipolar disorders patients agrupated in moderate and severe range hopeleness; the general population and the asmathics, hypertensives, tuberculous and $\mathrm{PBC}$ addicts patients agrupated in normal and mild range hopelessness. Concluding at the end that the BHS evaluated psychological significative construct and may to be used for screening.

Keywords: Beck Hopelessness Scale, Adaptation, Releability, Validity.

\section{INTRODUCCIÓN}

La depresión es uno de los desórdenes clínicos más frecuentes en la población general, existiendo diversos marcos conceptuales que partiendo de la práctica clínica y de los hallazgos de investigaciones intentan explicarla para intervenir sobre ella. Uno de estos modelos es el de Beck (1967) en el que su tercer componente denominado la tríada cognitiva consiste en (1) una visión negativa de si mismo; (2) una visión negativa del funcionamiento presente, y (3) una visión negativa del futuro. Este último aspecto es la desesperanza (pesimismo) que, conviene precisar, no sólo subyace a la depresión sino también a otros desórdenes psicológicos (Beck y Steer, 1988).

La medición del constructo desesperanza fue iniciada por Beck y sus colaboradores con la elaboración de la Escala de Desesperanza de Beck (Beck Hopelessness Scale, BHS) ciñéndose al modelo propuesto por Stotland (1969) quien la concibe como un sistema de esquemas cognitivos que tienen en común expectativas negativas acerca del futuro, sea este el inmediato o el más remoto. La persona desesperanzada cree: (1) que nunca podrá salir adelante por si mismo; (2) que nunca tendrá éxito en lo que ella intente; (3) que nunca podrá alcanzar objetivos importantes; y (4) que nunca podrá solucionar los diversos problemas que afronte en la vida.

El BHS no fue construido para detectar la desesperanza en adolescentes y en la población de adultos normales, sin embargo, como reporta el mismo Beck, se han realizado estudios con ambos propósitos e incluso con adolescentes de 13 años (Durham, 1986; Greene, 1981; Jonson y Cucheton, 1981; Tipol y Tezniikoff, 1982; citados en Beck y Steer, 1988). Por otro lado, el BHS ha mostrado consistentemente ser bastante útil como un indicador indirecto del riesgo de suicidio en depresivos o en individuos que han intentado el suicidarse (Beck y Steer, 1988). Esta particularidad y la posibilidad de su uso en la población general y en adolescentes, teniendo en cuenta que el $20 \%$ de limeños padecen de depresión la misma que esta cercanamente relacionada con el suicidio o intento de suicidio, cuya tendencia esta comprometiendo cada vez a adolescentes y niños (Vásquez, 1998); es lo que nos ha motivado a realizar su adaptación y ponerla a disposición de la comunidad de psicólogos para su utilización en la mediciòn de la desesperanza. En este contexto, el presente trabajo tiene por objetivos el adaptar y determinar las características psicométricas de confiabilidad y validez del BHS para evaluar la desesperanza. 


\section{MÉTODOS}

Sujetos. Los participantes conforman 7 grupos que hacen un total de 782 sujetos (335 varones y 447 mujeres).

- Grupo 1. 26 sujetos con por lo menos un intento de suicidio, que estaban recibiendo atención psicológica en centros de salud del Ministerio de Salud (Minsa).

- Grupo 2. 23 pacientes hospitalizados en un Hospital de Salud Mental de Lima, con diagnóstico de depresión mayor o trastorno bipolar

- Grupo 3. 68 pacientes entre hipertensos, asmáticos y tuberculosos que se atendían en varios centros de salud del Ministerio de Salud (Minsa).

- Grupo 4. 22 pacientes de un Hospital de Salud Mental con diagnósticos de esquizofrenia paranoide y trastornos esquizo-afectivos.

- Grupo 5. 25 pacientes dependientes a la pasta básica de cocaína (PBC) que recibían tratamiento en un centro especializado.

- Grupo 6. 61 estudiantes del 6to. grado de primaria de dos centros educativos de Lima Metropolitana.

- Grupo 7. 567 sujetos entre estudiantes del 5to de secundaria, madres de familia, estudiantes universitarios y docentes residentes en la capital, aparentemente sin trastorno mental alguno (Grupo Población General).

Una descripción más detallada de la muestra atendiendo al sexo, estado civil y grado educativo aparece en el siguiente cuadro 1.

Cuadro 1. Distribución de la muestra de estudio.

\begin{tabular}{|c|c|c|c|c|c|c|c|c|}
\hline & $\begin{array}{l}\text { Grupo 1 } \\
\text { Int. De } \\
\text { Suicidio }\end{array}$ & $\begin{array}{c}\text { Grupo } 2 \\
\text { Depresión } \\
\text { mayor/ } \\
\text { Trastorno } \\
\text { bipolar } \\
\end{array}$ & \begin{tabular}{|c|} 
Grupo 3 \\
Hipertensos, \\
asmáticos y \\
tuberculosos
\end{tabular} & $\begin{array}{c}\text { Grupo } 4 \\
\text { Esquizofrenia } \\
\text { paranoide } \\
\text { /Trastornos } \\
\text { afectivos. } \\
\end{array}$ & $\begin{array}{c}\text { Grupo 5 } \\
\text { Fármaco- } \\
\text { dependientes } \\
(\mathrm{PBC})\end{array}$ & $\begin{array}{c}\text { Grupo } 6 \\
\text { Est. de } \\
\text { primaria } \\
\text { (6to.grado } \\
\text { ) } \\
\end{array}$ & $\begin{array}{c}\text { Grupo } 7 \\
\text { Pob. } \\
\text { general }\end{array}$ & TOTAL \\
\hline Varón & $11(42 \%)$ & $11(48 \%)$ & $31(46 \%)$ & $22(100 \%)$ & $25(100 \%)$ & $21(41 \%)$ & $335(43 \%)$ & $335(43 \%)$ \\
\hline Mujer & $15(58 \%)$ & $12(52 \%)$ & $37(54 \%)$ & & & $30(59 \%)$ & $447(57 \%)$ & $447(57 \%)$ \\
\hline Solteros & $11(42 \%)$ & $17(74 \%)$ & $29(42 \%)$ & $9(41 \%)$ & $18(72 \%)$ & $51(100 \%)$ & $669(86 \%)$ & $669(86 \%)$ \\
\hline Casados & $15(58 \%)$ & $6(26 \%)$ & $38(56 \%)$ & $13(59 \%)$ & $5(20 \%)$ & & $109(14 \%)$ & $109(14 \%)$ \\
\hline Divorciados & $00(0 \%)$ & & $1(2 \%)$ & & $2(8 \%)$ & & $4(0 \%)$ & $4(0.5 \%)$ \\
\hline Ed. Primaria & $12(46 \%)$ & $7(30 \%)$ & $12(18 \%)$ & $6(27 \%)$ & $1(4 \%)$ & $51(100 \%)$ & $103(13 \%)$ & $103(13 \%)$ \\
\hline Ed. Secundaria & $10(39 \%)$ & $12(52 \%)$ & $32(47 \%)$ & $10(46 \%)$ & $12(48 \%)$ & & $362(46 \%)$ & $362(46 \%)$ \\
\hline Ed. Superior & $4(15 \%)$ & $4(18 \%)$ & $24(35 \%)$ & $6(27 \%)$ & $12(48 \%)$ & & $317(41 \%)$ & $317(41 \%)$ \\
\hline Edad (X) & 37.8 & 35 & 37.4 & 31.7 & 12.8 & 40.7 & 20 & 22.9 \\
\hline $\mathrm{N}$ & 26 & 23 & 68 & 22 & 25 & 51 & 567 & 782 \\
\hline
\end{tabular}

\section{Instrumento}

El BHS está basado en una visión cognitiva de la Desesperanza. Consta de 20 afirmaciones con dos opciones de respuesta, Verdadero o Falso. No tiene tiempo límite de aplicación, pero generalmente toma de 5 a 10 minutos. Los ítemes que indican desesperanza se califican con 1 punto; los que no la indican se califican con 0 puntos, de tal manera que el puntaje máximo es 20 y el mínimo es 0. 
El BHS sólo estima la severidad de la desesperanza, por ello es clínicamente importante prestar atención a otros aspectos del funcionamiento psicológico particularmente a los niveles de la depresión y la ideación suicida. Ayuda a la interpretación la siguiente escala de puntajes propuestas por Beck y Steer (1988): 0-3, Rango normal o asintomático; 4-8, Leve; 9-14, Moderado; y 15-20, Severo. Se ha observado que un puntaje mayor a 9 es un buen predictor de una eventual conducta suicida.

\section{Procedimiento}

1. Adaptación. Se realizó con la técnica back translation que hemos empleado anteriormente para la adaptación de otros cuestionarios (Aliaga, Giove y Dergan, 2003, y Aliaga, Giove, Dergan y Ponce, 2003). El BHS fue traducido del inglés al castellano por dos psicólogos peruanos que ejercen profesionalmente en USA*. Esta versión fue retraducida al inglés en Lima por un traductor profesional con la colaboración del autor principal, cotejándose a continuación ambas versiones en inglés para determinar su equivalencia, realizándose algunas ligeras modificaciones idiomáticas en la versión en español. Luego se administró el BHS a una muestra de 25 sujetos para evaluar la comprensión de las instrucciones y de los ítemes, después de unos pequeños reajustes la versión resultante quedó lista para su estudio psicométrico.

2. Administración de la prueba adaptada. Se administró a la muestra de estudio por psicólogos y estudiantes de psicología, cuidando de satisfacer las condiciones psicométricas usuales en esta actividad.

3. Análisis psicométrico. Ejecutado de acuerdo a la siguiente estrategia:

a. Para estimar el grado de capacidad discriminativa de cada item para diferenciar a los sujetos con alta y baja puntuación en el BHS se hizo un análisis de los ítemes obteniendo para cada uno de ellos mediante el coeficiente de correlación de Pearson su índice de discriminación o correlación ítem-total corregido ( $\mathrm{r}_{\mathrm{it}}$; ítem-remainder).

b. La confiabilidad del BHS fue estimada con dos técnicas: la consistencia interna utilizando el coeficiente alpha de Cronbach, y el test-retest usando el coeficiente de correlación de Pearson para correlacionar las puntuaciones del BHS obtenidas en dos momentos separados por un intervalo de administración de dos semanas.

c. Por otro lado, la validez del BHS fue estimada de tres maneras diferentes: primero, mediante la modalidad de la validez concurrente, correlacionando los puntajes del BHS *con los puntajes de los ítemes que evalúan el pesimismo en la escala de depresión de Beck (BDI), y **con esta escala a la que previamente se le extrajo los ítemes que evalúan el referido pesimismo. Segundo, mediante la modalidad de la validez factorial utilizando la técnica de los componentes principales y rotación varimax; tercero, mediante la modalidad de la validez diferencial determinando la relación del BHS con *el sexo y la edad por medio del coeficiente de correlación de Pearson, y**analizando mediante la prueba Ji cuadrado la distribución de la muestra en los niveles de desesperanza: normal o asintómatico, leve, moderado y severo.

\footnotetext{
* Agradecemos la colaboración del Psy. D. José Dergan Chaluja y del Ms. Alejandro Giove Percovich.
} 


\section{RESULTADOS}

1. El análisis de los ítemes arrojó la siguiente información:

Cuadro 2. Correlaciones ítem-total corregida por grupos.

\begin{tabular}{|c|c|c|c|c|c|c|c|c|}
\hline Ítem & $\begin{array}{c}\text { Grupo } 1 \\
\text { Int. de } \\
\text { suicidio }\end{array}$ & $\begin{array}{c}\text { Grupo 2 } \\
\text { Depresión } \\
\text { mayor/ } \\
\text { Trastorno } \\
\text { bipolar }\end{array}$ & $\begin{array}{c}\text { Grupo 3 } \\
\text { Hipertensos, } \\
\text { asmáticos y } \\
\text { tuberculosos }\end{array}$ & $\begin{array}{c}\text { Grupo } 4 \\
\text { Esquizofrenia } \\
\text { Paranoide } \\
\text { /Trastornos } \\
\text { afectivos } \\
\end{array}$ & $\begin{array}{c}\text { Grupo 5 } \\
\text { Fármaco- } \\
\text { dependientes } \\
\text { (PBC) }\end{array}$ & $\begin{array}{c}\text { Grupo 6 } \\
\text { Est. de } \\
\text { primaria } \\
\text { (6to. grado) }\end{array}$ & $\begin{array}{c}\text { Grupo } 7 \\
\text { Pob. } \\
\text { general }\end{array}$ & Total \\
\hline 1 & -0.09 & 0.66 & 0.11 & 0.81 & 0.61 & -0.13 & 0.37 & 0.42 \\
\hline 2 & 0.40 & 0.29 & 0.41 & 0.69 & 0.15 & 0.35 & 0.31 & 0.42 \\
\hline 3 & 0.39 & 0.13 & 0.03 & 0.30 & 0.66 & -0.04 & 0.11 & 0.19 \\
\hline 4 & -0.24 & 0.57 & -0.12 & 0.29 & 0.19 & -0.42 & 0.21 & 0.17 \\
\hline 5 & 0.08 & 0.35 & 0.03 & 0.81 & 0.19 & 0.07 & 0.21 & 0.22 \\
\hline 6 & 0.14 & 0.38 & 0.30 & 0.54 & 0.44 & 0.32 & 0.37 & 0.43 \\
\hline 7 & 0.23 & 0.67 & 0.38 & 0.75 & 0.72 & 0.18 & 0.35 & 0.44 \\
\hline 8 & -0.06 & 0.16 & -0.06 & 0.28 & 0.05 & 0.01 & -0.04 & 0.04 \\
\hline 9 & 0.17 & 0.52 & 0.22 & 0.66 & -0.10 & 0.42 & 0.23 & 0.33 \\
\hline 10 & 0.08 & 0.43 & 0.26 & 0.64 & -0.24 & 0.26 & 0.29 & 0.36 \\
\hline 11 & 0.50 & 0.24 & 0.25 & 0.93 & 0.56 & -0.24 & 0.34 & 0.44 \\
\hline 12 & 0.05 & 0.42 & 0.37 & 0.82 & 0.06 & 0.13 & 0.33 & 0.37 \\
\hline 13 & 0.03 & 0.04 & 0.36 & 0.35 & 0.75 & -0.13 & 0.23 & 0.25 \\
\hline 14 & 0.22 & -0.02 & 0.23 & 0.64 & 0.26 & 0.23 & 0.23 & 0.26 \\
\hline 15 & 0.21 & 0.61 & 0.40 & 0.79 & 0.40 & -0.08 & 0.20 & 0.34 \\
\hline 16 & 0.35 & 0.35 & 0.35 & 0.80 & 0.15 & 0.23 & 0.47 & 0.50 \\
\hline 17 & 0.34 & 0.50 & 0.37 & 0.81 & 0.45 & 0.38 & 0.31 & 0.46 \\
\hline 18 & 0.39 & 0.59 & 0.44 & 0.63 & 0.40 & 0.10 & 0.38 & 0.44 \\
\hline 19 & -0.12 & 0.13 & 0.22 & 0.61 & 0.22 & -0.22 & 0.17 & 0.23 \\
\hline 20 & 0.49 & 0.66 & 0.27 & 0.74 & 0.41 & 0.09 & 0.36 & 0.44 \\
\hline$\alpha$ & 0.56 & 0.82 & 0.65 & 0.94 & 0.70 & 0.37 & 0.68 & 0.76 \\
\hline $\mathrm{N}$ & (26) & (23) & (68) & (22) & (25) & (51) & $(567)$ & (782) \\
\hline
\end{tabular}

Los índices de discriminación mostrados en el Cuadro 2 permiten observar como estos índices varían a través de las siete muestras analizadas, situación que también hallaron los autores del BHS (Beck y Steer,1988). Existen índices negativos e inferiores a +0.20 valor considerado como el mínimo aceptable (Ary, Jacobs y Razavieh, 1990), apareciendo éstos en mayor número en las muestras (1) intento de suicidio y en la (6) escolares del 6to. grado de primaria; además, salvo en la muestra (4) Esquizofrenia paranoide/Trastornos afectivos, el ítem 8 «Espero ser particularmente suertudo/a y obtener más de las cosas buenas de la vida, que la persona promedio», muestra índices de discriminación negativos o bajos en todas las muestras, incluso en el promedio total $\left(r_{i t}=0.04\right)$. Sin embargo, al considerar los índices de discriminación totales estimamos conveniente no eliminarlo de la escala adaptada, pues no se elevaría sensiblemente el coeficiente alfa $(\mathrm{a}=0.76)$.

2. Confiabilidad. Su establecimiento tiene por objetivo estimar el error existente en la medida y su indicador es el coeficiente de confiabilidad. En la penúltima línea del cuadro 2 se observan los valores de los coeficientes alpha de Crombach que estiman la consistencia interna del BHS en cada uno de los grupos y en la muestra total. El 
análisis de los coeficientes por grupo indica que el grupo número 4 (Esquizofrenia paranoide / Trastorno esquizo-afectivo) tiene el valor más elevado (0.94), calificado como muy alto, siguiéndole el grupo número 2 (Depresión mayor / Trastorno bipolar), con un valor alpha (0.82) tipificado como moderado; en tanto que el grupo número 5 (Estudiantes del sexto grado de primaria) tiene el valor alpha más bajo de todos los grupos, muy pobre, indicando que la medida de la desesperanza en este grupo esta muy afectada por el error, hallazgo que se halla en la misma dirección que el reportado por Durham (1982, en Beck y Steer, 1988) quien hallaba que la consistencia interna era más baja en las muestra de escolares. Estos hallazgos sugieren que el BHS no debe ser aplicado en sujetos de este grado educativo y edad (edad promedio $=12.8$ ); resultado que, a su vez, abona a favor de la recomendación hecha por Beck y Steer (1988) de aplicar el BHS a partir de los 17 años de edad (final de los estudios secundarios). También es de señalar que el valor alpha bajo del grupo 1 (intento de suicidio), en el que era de esperar un valor más elevado, probablemente esté relacionado con el hecho que los miembros de este grupo se encontraban recibiendo psicoterapia de índole cognitivo-conductual. Por otro lado, el coeficiente alfa total es de un tamaño (0.76) relativamente bajo (Castaños, 1975), pero usual en las pruebas que evalúan características de la personalidad. Al comparar los coeficientes hallados con los que comunican Beck y Steer (1988) que varían de 0.82 a 0.92, se encuentra que los nuestros, salvo los de los citados grupos 4 y 6, son una cuantía inferior Aquí es pertinente indicar que los coeficientes de consistencia interna de pruebas adaptadas a nuestro medio son en nuestra experiencia menores a los obtenidos en las muestras originales (Aliaga y Pecho, 2000; Aliaga, Ponce, Bernaola y Pecho, 2001; Aliaga, Giove y Dergan, 2003; Aliaga, Giove, Dergan y Ponce, 2003)

Por otro lado, en el cuadro 3 se observa el valor del coeficiente test-retest resultante de la aplicación del BHS a una muestra de 100 sujetos del quinto año de secundaria (50 varones, 50 mujeres), y de la readministración a ellos mismos después de dos semanas.

Cuadro 3. Test-retest.

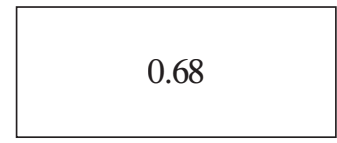

Este valor es estadísticamente muy significativo $(\mathrm{p}<0.01)$ al ser mayor que el $\mathrm{r}$ teórico $(0.05 ; 99)=0.205$; y en lo concerniente a su tamaño (size) es similar al de 0.69 de la escala original readministrada con un intervalo de seis semanas (Beck y Steer, 1988). Por lo demás estos valores se encuentran dentro del rango usual de los coeficientes de estabilidad de los tests de personalidad. En síntesis, este resultado así como el valor del coeficiente alpha de la muestra total ( 0.76 ; línea final del cuadro 2$)$ indican de acuerdo a los usos psicométricos que el BHS es una prueba con un grado de confiabilidad apreciable para el cumplimiento de sus propósitos, en especial en los grupos con características depresivas o de patología psicológica. 
3. Validez. La validez hace referencia a la pregunta ¿en qué grado la prueba mide el constructo que dice medir? Para dar una respuesta respecto del BHS se analizó su validez concurrente, validez factorial y validez diferencial.

Cuadro 4. Validez concurrente del BHS.

\begin{tabular}{|c|c|c|}
\cline { 2 - 3 } \multicolumn{1}{c|}{} & $\begin{array}{c}\text { BDI (solamente con } \\
\text { ítemes de pesimismo) }\end{array}$ & $\begin{array}{c}\text { BDI (sin ítemes de } \\
\text { pesimismo) }\end{array}$ \\
\hline BHS & $0.42^{* *}$ & $0.46^{* *}$ \\
\hline
\end{tabular}

** Correlación estadísticamente muy significativa $(p<0.001)$.

La correlación del BHS con el BDI tiene por resultado dos coeficientes estadísticamente muy significativos ( $\mathrm{p}<0.01$; una cola), lo que significa, respecto del primero, que el BHS mide en apreciable comunalidad la desesperanza (o pesimismo) que también evalúa el BDI; por otro lado, respecto del segundo, que la desesperanza evaluada por el BHS se relaciona con la depresión, cosa que era de esperar por ser una de las características principales de esta última, justificándose dicho sea de paso su inclusión en el modelo de la depresión de Beck (tríada cognitiva).

Por su parte, la validez factorial hace posible estimar la validez de constructo del BHS dando respuesta a la pregunta «¿qué proporción de la varianza de las puntuaciones de los sujetos que responden a la escala se puede atribuir a la variable que mide?». En la muestra total $(\mathrm{N}=782)$ se determinó previamente la idoneidad de la matriz de correlaciones de los ítems del BHS, para ser sometida al análisis factorial. El determinante fue de 0.012 , la medida de adecuación de muestreo $\mathrm{KMO}$, de 0.82 (meritorio) y el test de esfericidad de Bartlet tuvo un valor Ji cuadrado estadísticamente muy significativo $(\mathrm{p}<0.0001)$. Aplicado el análisis factorial con la técnica de los componentes principales y rotación varimax empleamos el criterio de retener al factor con un eigen value o valor propio igual o superior a 1.0; de esta manera se halló seis factores que subyacen al BHS y que explican en conjunto el $49.32 \%$ de la referida varianza, valor considerado psicométricamente como satisfactorio. Es de señalar que en un muestra de 294 sujetos con intento de suicidio se halló tres factores y en una muestra de 83 pacientes con depresión mayor, cinco componentes (Nekanda-Trepka, Bishop y Blackburn, 1982, en Beck y Steer, 1988). En el cuadro 5 se consigna la estructura factorial del BHS encontrado en la muestra peruana, indicándose los factores con sus ítemes correspondientes y las saturaciones o cargas de éstos, las que por ser, en casi todos los casos de 0.50 o más propician la clara definición e interpretación de los factores (Comrey, 1985). 
Cuadro 5. Estructura factorial del BHS.

\begin{tabular}{|c|c|c|c|c|c|c|}
\hline Ítem & $\begin{array}{c}\text { Factor 1 } \\
\text { Carencia de } \\
\text { ilusión en el } \\
\text { futuro }\end{array}$ & $\begin{array}{c}\text { Factor } 2 \\
\text { Expectativa } \\
\text { de infelicidad } \\
\text { en el futuro }\end{array}$ & $\begin{array}{c}\text { Factor } 3 \\
\text { Expectativa } \\
\text { de desgracia } \\
\text { en el futuro }\end{array}$ & $\begin{array}{c}\text { Factor } 4 \\
\text { Expectativa } \\
\text { de un futuro } \\
\text { incierto }\end{array}$ & $\begin{array}{c}\text { Factor } 5 \\
\text { Creencia que los } \\
\text { acontecimientos } \\
\text { negativos son } \\
\text { durables } \\
\end{array}$ & $\begin{array}{c}\text { Factor } 6 \\
\text { Creencia en la } \\
\text { imposibilidad de } \\
\text { ser favorecido } \\
\text { por el azar }\end{array}$ \\
\hline 6 & 0.52 & & & & & \\
\hline 7 & 0.46 & & & & & \\
\hline 9 & 0.66 & & & & & \\
\hline 12 & 0.55 & & & & & \\
\hline 16 & 0.59 & & & & & \\
\hline 17 & 0.68 & & & & & \\
\hline 20 & 0.62 & & & & & \\
\hline 5 & & 0.63 & & & & \\
\hline 10 & & 0.49 & & & & \\
\hline 14 & & 0.58 & & & & \\
\hline 15 & & 0.52 & & & & \\
\hline 13 & & & 0.62 & & & \\
\hline 19 & & & 0.70 & & & \\
\hline 1 & & & & 0.50 & & \\
\hline 4 & & & & 0.80 & & \\
\hline 18 & & & & 0.70 & & \\
\hline 3 & & & & & 0.80 & \\
\hline 8 & & & & & & 0.83 \\
\hline
\end{tabular}

En cuanto a la validez diferencial, ésta fue evaluada para determinar la eficacia del BHS para apreciar diferencias en la manifestación de la desesperanza con relación a dos variables demográficas importantes en este contexto: el sexo y la edad; y para distinguir a grupos en lo que es esperable la desesperanza de aquellos en lo que no es esperable esta.

En el cuadro 6 se muestran los valores de los coeficientes $r$ de Pearson del BHS con el sexo y la edad, y en el cuadro 7 la distribución porcentual de la muestra en los niveles de desesperanza (Beck y Steer, 1988) con el valor de la prueba Ji cuadrado de homogeneidad utilizado para averiguar la significatividad estadística de las diferencias en esta distribución.

\section{Cuadro 6}

\section{* Correlación estadísticamente muy significativa $(p<0.01)$.}

En el cuadro 6 se observa que no existe correlación entre el sexo y la desesperanza; y que la desesperanza está positivamente relacionada con la edad ( $\mathrm{p}<0.01)$, sin embargo esta última correlación siendo significativa es relativamente baja (Elorza,1987), solamente el $9 \%$ de la variabilidad en los puntajes de BHS pueden ser atribuidos a la edad. Similares resultados encontró Greene (1981) en una muestra de 396 adultos irlandeses. Pareciera entonces que el hecho de ser varón o mujer no implica el padecer 
más o menos desesperanza, y que existe una tendencia significativa pero leve (Elorza, 1987) a que al aumentar la edad aumenta la desesperanza y viceversa. Por otro lado, estos resultados hacen innecesario el ajuste de las puntuaciones en la BHS según el sexo y la edad.

Cuadro 7. Distribución de la muestra por niveles de desesperanza.

\begin{tabular}{|c|c|c|c|c|c|}
\hline \multicolumn{6}{|c|}{ NIVELES DE DESESPERANZA } \\
\hline & $\begin{array}{c}(0-3) \\
\text { Normal/ } \\
\text { Asintomático }\end{array}$ & $\begin{array}{l}(4-8) \\
\text { Leve }\end{array}$ & $\begin{array}{c}(9-14) \\
\text { Moderado }\end{array}$ & $\begin{array}{l}(15-20) \\
\text { Severo }\end{array}$ & TOTAL \\
\hline $\begin{array}{l}\text { Grupo } 1 \\
\text { Intento de suicidio }\end{array}$ & $\begin{array}{c}4 \\
(15.4 \%)\end{array}$ & $\begin{array}{c}15 \\
(57.7 \%)\end{array}$ & $\begin{array}{c}7 \\
(26.9 \%)\end{array}$ & $\begin{array}{c}0 \\
(0 \%)\end{array}$ & 26 \\
\hline $\begin{array}{l}\text { Grupo } 2 \\
\text { Depresión mayor/ } \\
\text { Trastorno bipolar }\end{array}$ & $\begin{array}{c}1 \\
(4.3 \%\end{array}$ & $\begin{array}{c}5 \\
(21.7 \%)\end{array}$ & $\begin{array}{c}10 \\
(43.5 \%)\end{array}$ & $\begin{array}{c}7 \\
(30.4 \%)\end{array}$ & 23 \\
\hline $\begin{array}{l}\text { Grupo } 3 \\
\text { Hipertensos, asmáticos y } \\
\text { tuberculosos }\end{array}$ & $\begin{array}{c}26 \\
(38.2 \%)\end{array}$ & $\begin{array}{c}36 \\
(52.9 \%)\end{array}$ & $\begin{array}{c}6 \\
(8.8 \%)\end{array}$ & $\begin{array}{c}0 \\
(0 \%)\end{array}$ & 68 \\
\hline $\begin{array}{l}\text { Grupo } 4 \\
\text { Esquizofrenia paranoide/ } \\
\text { Trastornos afectivos }\end{array}$ & $\begin{array}{c}9 \\
(40.9 \%)\end{array}$ & $\begin{array}{c}6 \\
(27.3 \%)\end{array}$ & $\begin{array}{c}1 \\
(4.5 \%)\end{array}$ & $\begin{array}{c}6 \\
(27.3 \%)\end{array}$ & 22 \\
\hline $\begin{array}{l}\text { Grupo } 5 \\
\text { Farmacodependientes a la } \\
\text { PBC }\end{array}$ & $\begin{array}{c}14 \\
(56 \%)\end{array}$ & $\begin{array}{c}10 \\
(40 \%)\end{array}$ & $\begin{array}{c}0 \\
(0 \%)\end{array}$ & $\begin{array}{c}1 \\
(4 \%)\end{array}$ & 25 \\
\hline $\begin{array}{l}\text { Grupo } 6 \\
\text { Estudiantes del 6to. de } \\
\text { primaria }\end{array}$ & $\begin{array}{c}8 \\
(40.9 \%)\end{array}$ & $\begin{array}{c}35 \\
(27.3 \%)\end{array}$ & $\begin{array}{c}8 \\
(4.5 \%)\end{array}$ & $\begin{array}{c}0 \\
(0 \%)\end{array}$ & 51 \\
\hline $\begin{array}{l}\text { Grupo } 7 \\
\text { Población general }\end{array}$ & $\begin{array}{c}321 \\
(56.6 \%)\end{array}$ & $\begin{array}{c}207 \\
(36.5 \%)\end{array}$ & $\begin{array}{c}37 \\
(6.5 \%)\end{array}$ & $\begin{array}{c}2 \\
(0.4 \%)\end{array}$ & 567 \\
\hline TOTAL & $\begin{array}{c}383 \\
(49 \%)\end{array}$ & $\begin{array}{c}314 \\
(40.2 \%)\end{array}$ & $\begin{array}{c}69 \\
(8.8 \%)\end{array}$ & $\begin{array}{c}16 \\
(2 \%)\end{array}$ & $\begin{array}{c}782 \\
(100 \%)\end{array}$ \\
\hline Ji cuadrado & $\begin{array}{c}1519.15 \\
(\mathrm{p}<0.0001)\end{array}$ & $\begin{array}{c}706.0 \\
(p<0.0001)\end{array}$ & $\begin{array}{c}71.703 \\
(\mathrm{p}<0.0001)\end{array}$ & $(\mathrm{p}<0.09)$ & \\
\hline
\end{tabular}

En el cuadro 7 se observan tres cosas muy importantes respecto a la validez de constructo:

a. La prueba Ji cuadrado de homogeneidad alcanza valores estadísticamente muy significativos en las categorías o niveles de desesperanza normal/asintomático, leve y moderado, pero no en la categoría severo en la que el valor de esta prueba es estadísticamente marginal (0.09). La revisión de las suposiciones del modelo de esta prueba estadística indica que no se ha cumplido con una de ellas, siendo el resultado espurio. En efecto, cuando los grados de libertad son mayores de 1, no más del $20 \%$ de las frecuencias esperadas o efectivos calculados pueden estar debajo de 5 (Clark-Carter, 2000). En la categoría severo hay 5 grados de libertad siendo las frecuencias esperadas para cada uno de los grupos de 2.28 (datos no consignados en el cuadro) estando todas por debajo de 5. En consecuencia, atendiendo a los grupos con porcentajes más elevados pasamos a determinar si habían diferencias entre los porcentajes de los grupos 2 (30.49\%) y el grupo 4 (27.39\%). De acuerdo a la fórmula: para que existan diferencias significativas, el doble del porcentaje menor no debe superar al porcentaje mayor comparado, encontramos lo siguiente: 27.39 × $2=54.78<30.49$; por tanto, las diferencias porcentuales observadas se deben al azar, a la casualidad, es decir, ambos grupos $(2=$ Depresión 
mayor/trastorno bipolar; 4 = Esquizofrenia paranoide/Trastornos afectivos) no difieren significativamente en porcentajes en la categoría severo.

b. En la categoría severo se ubica el $2 \%$ de la muestra total $(\mathrm{N}=782)$, correspondiendo de este total como hemos visto el $30.4 \%$ al grupo 2 y el $27.3 \%$ al grupo 4 , en tanto que sólo el $0.4 \%$ de los sujetos del grupo población general se halla en esta categoría.

c. Unido con lo anterior se observa que en la categoría moderado se halla el $43.5 \%$ del grupo 2 (Depresión mayor/trastorno bipolar), el 26.3\% del grupo 1 (Intento de suicidio), y sólo un $6.5 \%$ del grupo Población general. En otros términos, 7 de cada 10 sujetos del grupo $2(30.4 \%+43.5 \%=73.9 \%)$ se ubican en las categorías moderado/severo de desesperanza, en tanto que 9 de cada 10 sujetos del grupo Población general $(56.6 \%+36.5 \%=93.1 \%)$ se ubican en las categorías normal/ leve, distribución igual a la de los grupos 3 (Hipertensos, asmáticos, TBC) y 5 (Farmacodependientes a la PBC), este último resultado sugiere que la desesperanza no es una característica de este tipo de pacientes. Por otra parte, llama la atención que en el grupo 1 (Intento de suicidio) ningún sujeto se ubique en la categoría severo mientras que 8 de cada 10 lo hagan en las categorías leve/moderado. Como dijimos más arriba es probable que esta distribución se encuentre relacionada con el hecho que estos sujetos estaban sometidos a una terapia de tipo cognitivo-conductual.

En síntesis, el análisis de estos resultados permite afirmar que la escala BHS adaptada muestra evidencias de tener validez de constructo.

\section{CONCLUSIONES}

1 Se logra la adaptación de la Escala de Desesperanza de Beck (BHS) para su uso en la población de Lima Metropolitana

2 La Escala de Desesperanza de Beck (BHS) adaptada tiene índices de confiabilidad y validez que la hacen un instrumento útil para el screening de la desesperanza o pesimismo.

\section{REFERENCIAS BIBLIOGRÁFICAS}

1. Aliaga, J.,Giove, A. y Dergan, J.(2003). Adaptación y características psicométricas del MSLQ. Ponencia presentada en el XXIX Congreso Interamericano de Psicología, Lima.

2. Aliaga, J.,Giove, A., Dergan, J. y Ponce, C. (2003). Inventario de estrategias de estudio y aprendizaje (LASSI): Adaptación, estructura factorial, validez y baremos. Ponencia presentada en el XXIX Congreso Interamericano de Psicología, Lima.

3. Aliaga, J., Ponce, C., Bernaola, E. y Pecho, J. (2001). «Características psicométricas del inventario de autoevaluación de la ansiedad ante exámenes». En: Paradigmas, Revista del Colegio de Psicólogos del Perú, Año 2, Vol 2, No 3-4: 11-30.

4. Aliaga, J.y Pecho, J.(2000). «Evaluación de la actitud hacia la matemática en estudiantes secundarios». En: Paradigmas, Revista del Colegio de Psicólogos del Perú, Año 1, Vol 1, No 1-2: 61-78. 
5. Ary, D., Jacobs, L.C. y Razavieh, A. (1990). Introduction to research in education ( $4^{\text {th }}$. ed.). Chicago: Hold, Rinehart and Winston.

6. Beck, A. y Steer, R. (1988). Beck Hopelessness Scale. Manual. New York: The Psychological Corporation Harcourt Brace Jovanovich, Inc.

7. Beck, A. (1967). Depression: Causes and treatment. Philadelphia: University of Pennsylvania Press.

8. Clark-Carter, D.(2002). Investigación cuantitativa en psicología. México: Oxford.

9. Castaños, C.(1975). «Selección de los elementos de una Escala de Personalidad». Revista de Psicología General y Aplicada. № 130, Vol XXX: 609-618-

10. Comrey, A. (1985). Manual de análisis factorial. Madrid: Cátedra.

11. Elorza, H. (1987). Estadística para ciencias del comportamiento. México: Harla

12. Greene, S.M. (1981). Levels of measured hopelessness in the general population. British Journal of Clinical Psychology, 20, 11-14.

13. Scotland, E. (1969). The psychology of hope. San Francisco: Jossey Bass.

14. Vásquez, F. (1998). «Seguimiento de pacientes que presentaron intento de suicidio atendidos en el servicio de emergencia del INSM Honorio Delgado - Hydeyo Noguchi (1996 - 1998)». Anales de Salud Mental. Instituto Nacional de Salud Mental «Honorio Delgado-Hydeyo Noguchi», Lima. Vol. XIV, Nos. 1 y 2:65-76. 
